\title{
EIN NEUER BELEG ZUM PHRYGISCHEN URSPRUNG DER ARCHITEKTONISCHEN TONPLATTEN
}

Fahri IŞIK

\begin{abstract}
Schlüsselwörter: Phryg $\bullet$ Tonplatten $\bullet$ Früharchaik $\bullet$ Theseus-Minotauros $\bullet$ Bey-Boğaadam Anahtar Kelimeler: Frig • pişmiştoprak levhalar • Erken Arkaik • Theseus-Minotauros • Bey-Boğaadam.
\end{abstract}

\section{Özet:}

Ege dünyasının sevilerek uygulanan mimari süslemelerinden pişmiş toprak levhaların bilinen en erken örnekleri Frig başkenti Gordion'dandır. Geometrik bezekli olanların, Midas Çağı'na tarihlenen bir Yazılıkaya Kaya Tapınağı alnacında aynısıyla kabartma çerçeve biçiminde varlığı, bu yaratıcılığın ilk somut tanıtı olmalıdır; çünkü E. Akurgal'ın doğru saptamasıyla, kaya anıtlarında "okunan aynı bezeme diline, onlarla eşzaman Frig terrakotta frizleri de sahiptir". Yine Yassıhöyük'te aynı megaronun alnacını bezeyen geyik avı ve beyboğaadam mücadelesi, Yenihitit orthostatları üzerinde vardır; yan yana dizili levhalar düzeninde bir önemli yapı cephesini süslemeleri de işlevde birbirlerinden farklı değildir. Kökenin Doğululuğu, avda gözlemlenen durağanlıkta ve bey gücünün öne çıkarılmasında da okunur ve bunlarla ulaşılan en geç M.Ö. 7. yüzyıl başları tarihi zaman biçemine de uyar; çünkü gelişim, izleyen M.Ö. 650-550 arası süreçte dört kümede irdelenebilen Pazarlı savaşçı levhalarıyla sürecektir. AnatSt 1991'de kapsamlı biçim ve biçem gelişimiyle ulaştığım bu sonuç, yakın geçmişte ele geçen ve Arkeoatlas 2006'da tanıtılan bir Gordion buluntusuyla doğrulanmaktadır. Küçük fildişi kakma üzerine işli bu savaş̧̧ resminin K. DeVries tarafından iyi bir gözlemle varılan "M.Ö. 7. yüzyıl sonları" tarihi, Gordion ve Pazarlı pişmiş toprak yapı levhaları için öngördügüm erken kronolojinin de belgesi niteliğindedir. Çünkü bu yeni Gordion savaşçı betisi, boğaadamla mücadele eden beyin biçemini aynı sanat merkezinde açık ara geliştiren ileri özellikler içerir ve bu özellikleriyle yerini Pazarlı savaşçıları arasında 1. kümeden 2. kümeye geçiş evresinde bulur. Ve sonuçta Hellenleştirilerek 150 yıl gibi geç bir zamana tarihlenen ve de "Theseus-Minotauros" olarak yorumlanan başkent ürünü bir Frig resminin, aslında Doğu'nun sevilen "Bey-Boğaadam" mücadelesini betimlediği ve de -tıpkı "HeraklesNemea Aslanı" kümesinde gözlemlendiği gibi- Hellen dünyasında bir tanınmış mitosa uyarlandığ gerçeği de anlaşılmış olur. 
Die in der Archäologie einstimmig akzeptierte These, wonach die Phryger die Tradition der tönernen Verkleidungsplatten über Lydien von Aiolis übernommen habe ${ }^{1}$, wurde durch meinen Beitrag mit dem Titel "Zur Entstehung der tönernen Verkleidungsplatten in Anatolien" widerlegt. Als Folge der formalen, bildmotivischen und stilistischen Untersuchungen kam ich dort zum Schluss, dass die Einflusswelle in die umgekehrte Richtung, nämlich vom mittelanatolischen Hochland an die westanatolische Küste rollte und darin die Phryger den gebenden und die Aiolier bzw. Lyder den nehmenden Teil bildeten. Da diese architektonischen Formen mit den zeitgenössischen Orthostatenreliefs, die sogar in Gordion selbst in neuhethitischer Art und mit denselben Bildthemen Verwendung fan$\mathrm{den}^{3}$, funktional, formal, thematisch und bildmotivisch identisch sind, dürften sie von dieser beliebten Gattung der staatlichen Bauten Südostanatoliens abgeleitet und auf die Wände ihrer königlichen Bauten von Yassıhöyük in Ton übertragen worden sein.

Ausserdem ist in der Forschung längst erkannt, dass die in die Fassadenfläche

Dieser kurze Beitrag bildet eine Zusammenfassung meines in der Festschrift für M. Darga (2008) unter dem Titel „Pişmiş Toprak Mimari Levhaların Frig Kökenine Yeni Bir Kanıt" erschienenen Aufsatzes.

1 Bittel 1939, 143; Schefold 1950, 137 ff.; Akurgal 1955, 69 ff.; Akerström 1966, 216 ff. 229; Prayon 1987,174 ff.; DeVries 2006b, 60.

2 Işık 1991, 63-86.

3 Sams 1989, 447-454; Sams 1994, 211-220. Zum neuhethitischen Einfluss auf Orthostaten in Gordion s. Voigt 2006, 31; vor allem Strobel 2008, 139 mit Literatur. der Felstempel Phrygiens eingehauenen Quadrate "als eine Imitation der in Gordion und Pazarlı angetroffenen tönernen Verkleidungsplatten aufzufassen sind und diese sicher eine Sitte der zeitgenössischen Architektur widerspiegeln"4. Die berühmteste Kultfassade Yazllkaya in der Midasstadt mit geometrischem Dekor ist zu Beginn der phrygischen Felsdenkmäler unter dem urartäischen Einfluss ebenfalls eine Schöpfung der blühenden Midaszeit gewesen ${ }^{5}$. In der Forschung wird versucht, dieses Problem durch die Datierung der frühen Felskultmäler bzw. Kybelereliefs in die späte Phase der phrygischen Kunst, nämlich innerhalb des 6. Jahrhunderts v. Chr., zu lösen, wodurch aber das ganze 7. Jahrhundert im Dunkeln bleibt und damit zusammenhängend ein innerhalb dieser Zeitspanne gesuchter Vermittler für die vielseitigen Beziehungen zwischen der neuhethitischen und der ionischen Kunst und Kultur in der goldenen Epoche fehlt ${ }^{6}$.

In meinem Aufsatz über die Entstehung der tönernen Verkleidungsplatten in AnatSt 1991 stellte ich ausserdem fest, dass nicht in dem von einflussreichen Kunstzentren weit abgele-

Akerström 1966, 156; Akurgal 1955, 92; vgl. Iş1k 1991, 63 f. Taf. 9a (Kultdenkmal von Bahşı̧) mit Taf. 9b (Tonplatte von Pazarli).

5 Işı1k 1991, 64. Zur Fassade des Midas s. zuletzt Tüfekçi Sivas 1999, 52 ff. Taf. 9-23; Berndt 2002, 9 ff. Abb. 1. 8-16.

6 Dazu ausführlich s. Iş1k 1986 Beibl. 41-108; Roller 1999, 44 ff. 53 ff.; Tüfekçi Sivas 1999, 204 ff.; Berndt 2002, 14. 
genen Pazarl1, sondern in Gordion, der blühenden Hauptstadt des Midas, mit ihren bisher bekannten frühesten Tonplatten, der Ursprung dieser Gattung zu finden ist. Auch diese sind in der Forschung nicht aus stilistischen Gründen, sondern nach dem -wohl nicht gesicherten- stratigraphischen Befund zum Einen erst nach der persischen Invasion bzw. nach 550 v. Chr., zum Anderen aber schon um 600 v. Chr. datiert worden ${ }^{7}$; das dafür vorgeschlagenen letzte Datum liegt dazwischen, nämlich zwischen " 575 und 550" v. Chr.". Aber wenn man bedenkt, dass die Datierung der Zerstörungsschicht von Yassihöyük neuerdings rund 100 Jahre früher, also "an das Ende des 9. Jahrhunderts v. Chr.”, angesetzt wird ${ }^{9}$, und wenn diese, die Kultur- und Kunstgeschichte Anatoliens in der frühen Eisenzeit völlig verändernde Neudatierung richtig ist, dürften sowohl der frühere Datierungsversuch der Platten nach dieser Methode sogar "um 600 v. Chr." und damit zusammenhängend auch die Annahme über ihre Entstehung unter dem "griechischen" Einfluss noch fragwürdiger sein.

Von den von mir stilistisch und bildmotivisch -wohl spätestens- in die erste Hälfte des 7. Jahrhunderts v. Chr. datierten hauptastädisch-phrygischen Tonplatten werden hier nur diejenigen mit Herrscher und Stiermensch angesprochen

Dazu s. Prayon 1987, 179.

DeVries 2006b, 60.

9 Voigt 2006, 31 f.; DeVries 2006a, 44; DeVries 2006b, 58; vor allem Strobel 2008, 137 ff.
(Abb. 1. 2) ${ }^{10}$, deren Datstellungen bildmotivisch mit dem für die Entstehungsfrage dieser Gattung entscheidenden Neufund aus demselben Ort gut vergleichbar sind: Bei ihm handelt es sich um einen auf einer kleinen Elfenbeinplatte dargestellten Krieger, der von $\mathrm{K}$. DeVries trefflich an das Ende des 7. Jahrhunderts v. Chr. datiert wurde (Abb. 5) ${ }^{11}$. Er stimmt in der knienden Haltung mit dem Stiermensch, in der Form des kurzen Schwerts mit dem Herrscher und im Gewandmotiv mit Stiermensch und Herrscher auf den tönernen Verkleidungsplatten überein. DeVries erklärt die Darstellungen auf diesen beiden Platten aus verschiedenen Materialien bildmotivisch für östlich, gewandmotivisch aber für "griechisch" bzw. korinthisch; auch im Stil sollen beide dem "griechischen" treu geblieben sein. Ebenso stelle die -nach ihr- "Theseus-Minotauros"-Platte ein griechisches Thema dar, das über Lydien nach Gordion übertragen worden sei; und diese tönerne Platte soll rund ein halbes Jahrhundert später entstanden sein als die elfenbeinerne; nämlich in der Zeit zwischen " 575 und 550 v. Chr." 12 .

Als Folge dieser traditionsgebundenen Gemeinsamkeiten können die unverkennbaren stilistischen Unterschiede zwischen den beiden Werken aus dem-

\footnotetext{
10 Iş1k 1991, 82 ff. Taf. V (mit Literatur).

11 DeVries 2006a, 48 mit Abb; DeVries 2006b, 59; Karul 2006, auf S. 6.

12 De Vries 2006b, 60.
} 
selben Ort und demselben Kulturkreis nur zeitlich erklärt werden ${ }^{13}$. So zeigen die Figuren auf der HerrscherStiermensch-Platte aus Ton, verglichen mit dem Krieger auf der Elfenbeinplatte, bis in alle Einzelheiten, also in Haltung, Proportionierung, Körper-, Kopf- und Haarbildung, in den Gesichtszügen, sowie in der Form des Schwertsgriffes und Art des Schwertshaltens sehr augenfällige Unterschiede, was darauf hinweist, dass die Tonplatte zweifelsohne mit Abstand früher entstanden sein muss. Sogar die Krieger auf den von mir später als diese Tonplatte, nämlich in die zweite Hälfte des 7. Jahrhunderts v. Chr. datierten Verkleidungsplatten der "Gruppe I" von $\mathrm{Pa}-$ zarlı (Abb. 3. 4) ${ }^{14}$, können in ihren Formen und stilistisch nur eine unmittelbare Vorstufe für den Elfenbeinkrieger von Gordion bilden. Ein letzter Vergleich dieser knienden Krieger mit den PazarlKriegern der "Gruppe II" (Abb. 6) ${ }^{15}$, die meiner stilistischen Gruppenbildung nach aus der Übergangszeit vom 7. zum 6. Jahrhundert v. Chr. stammen dürften, zeigt, dass die Figur auf der gordischen Elfenbeinplatte ihren Platz stilistisch zwischen den Kriegern mit Lanzen der "Gruppe I" und der "Gruppe II" von Pazarlı finden $\mathrm{kann}^{16}$; also genau in der Stil-

13 Dazu s. ausführlich Işık 2008, 278 ff.

14 Iş1k 1991, 66 Abb. 1 „Gruppe Ia-c“, Taf. 10a-c; Akurgal 1955, Taf. 45a. b; Akerström 1966 Taf. 90, 1. 2; 91,1; DeVries 2006a, Abb. auf s. 42 o.; 45 u.

15 Iş1k 1991, 67 Abb. 1 "Gruppe II", Taf 11a. b; Akurgal 1955, Taf. 46b.

16 Aus dem Vergleich mit den Vertretern der beiden ersten Gruppen von Pazarlı (Işı1k 1991, 71 f.; hier Abb. 3. 4. und 6) geht deutlich hervor, dass die stufe, die für die elfenbeinerne Figur von DeVries selbst festgelegt wurde, nämlich "am Ende des 7. Jahrhunderts v. Chr."

Am Ende dieser Stilentwicklung stehen die -auch in der Forschung wegen der eindeutig entwickelteren Formen zeitlich für "spät" erklärten- Pazarl1Krieger auf den Platten der "Gruppe IV" (Abb. 7. 8) ${ }^{18}$, womit ich den Zeitstil des zweiten Viertels des 6. Jahrhunderts v. Chr. zu erreichen glaubte ${ }^{19}$. So sind sie die Schöpfungen einer Epoche der phrygischen Kunst gewesen, in der nach DeVries auch die Figuren der sog. "Theseus-Minotauros"Platten von Gordion entstanden sein sollten $^{20}$. Bei den Pazarl1-Kriegern ist

Platten mit Hirschjagd, Herrscher und Stiermensch aus Yassıhöyük (Işık 1991, 82 ff.; hier Abb. 1. 2) ihren Platz eher an der beginnenden Phase des 7. Jahrhunderts v. Chr. finden dürften. Ein Vergleich der Kriegerdarstellungen auf den Platten der Gruppe I von Pazarl (hier Abb. 3. 4) mit dem Krieger auf der Elfenbeinplatte von Yassıhöyük (hier Abb. 5) weist ebenfalls deutlich darauf hin, dass die ersteren zeitlich kaum bis ans Ende des 7. Jahrhunderts hinabreichen könnten.

17 DeVries 2006a, 48; DeVries 2006b, 59.

18 Iş1k 1991, 68 ff. Abb. 1 "Gruppe IV" Taf. 13a-c; Akurgal 1955, 74 ff. Taf. 48a.b. 49a. 50; Akerström 1966, 167 ff. Taf. 95. 96; Prayon 1987, 174 ff. Kat. Nr. 206 Taf. $38 f$.

19 Iş1k 1991, 76.

20 DeVries 2006b, 59 f. Auch Wittke 2006, 14, hält dieses Bildmotiv als Beleg für die Beziehungen zwischen den Griechen und den Phrygern und bezeichnet es als "Theseus-Minotauros-Gruppe". $\mathrm{Da}$ die frühen Bilder der Theseus-MinotaurosGruppe in der griechischen Kunst erst später als die Herrscher-Stiermensch-Gruppe von Gordion stammen, Schefold 1964, 37 Abb. 7; Akurgal 1966, 188 Fig. 121, ist nicht darauf zu zweifeln dass dieses Kampfschema nur eine griechische 
aber die Stilentwicklung zu natürlicheren Formen an allen Teilen und einzelnen Zügen so stark ablesbar, dass ihre Gleichzeitigkeit mit dem bekannten Gruppenbild von Gordion nicht möglich ist. Wenn nach DeVries die Künstler bei den Darstellungen auf den "in Gordion gefundenen lokalen Verkleidungsplatten dem -wohl ionisch bzw. anatolisch gemeinten- griechischen Stil treu blieben" ${ }^{21}$ und die ionische Meisterhand auch an den Kriegern von einem abgelegenen Ort wie Pazarlı unverkennbar ist ${ }^{22}$, dann können die auf den ersten Blick wahrnehmbaren Unterschiede zwischen den gattungsmässig und handwerklich miteinander identischen Werken desselben Kulturkreises nur zeitlich bedingt gewesen sein; den in meinem alten Beitrag in AnatSt. 1991 gebildeten Entwicklungsreihen nach dürfte dieser Unterschied zeitlich wohl nicht weniger als 100 Jahre gewesen sein.

So dürfte DeVries durch ihre eigene Datierung der Kriegerfigur auf der Elfenbeinplatte "ins späte 7. Jahrhunderts v. Chr." ${ }^{23}$ meine Thesen nachdrücklich bestätigt haben; nämlich: die Entstehung ei-

Umsetzung des östlichen Kampfmotives sein kann. So ist von Akurgal trefflich auch das beliebte griechische Bildschema des "Herakles mit dem nemäischen Löwen” interpretiert worden, Akurgal, ebenda mit Fig. 120. Zu einem spätgeometrischen Bild auf einem tönernen Dreifuss aus Kerameikos mit demselben, ursprünglich orientalischen Kampfmotiv s. Schefold 964, 20 Taf. 5a.

${ }^{21}$ DeVries 2006b, 60.

${ }^{22}$ Işık 1991, $68 \mathrm{ff}$.

23 DeVries 2006a, 48; DeVries 2006b, 60. ner in der Aegäis zukunftsträchtigen Gattung in der phrygischen Hauptstadt und die Richtigkeit der mit den gordischen Pionierstücken um 700 v. Chr. begonnenen und durch die Funde von Pazarlı bis 550 v. Chr. in vier Gruppen fortlaufenden Entwicklungsreihen.

Prof. Dr. Fahri Işık

Akdeniz Üniversitesi

Fen Edebiyat Fakültesi

Arkeoloji Bölümü

TR - 07058 Antalya 


\section{Abbildungsnachweis}

Abb. 1. İstanbul, Museum. Tönerne Herrscher-Stiermensch-Platte aus Gordion. Rekonstruktion (Akerström 1966, Taf. 79).

Abb. 2. Ankara, Museum. Fragmentiertes Herrscherbild aus der Gruppe in Abb. 1 (Işs $1 \mathrm{k}$ 1991, Taf. 15b).

Abb. 3. Ankara, Museum. Tönerne KriegerPlatte der "Gr. Ia” aus Pazarlı (Işı1k 1991, Taf. 10a).

Abb. 4. Ankara, Museum. Tönerne KriegerPlatte der "Gruppe Ic" aus Pazarlı (Işık 1991, Taf. 10c).
Abb. 5. Gordion, Museum. Elfenbeinerne Krieger-Platte aus Gordion (DeVries 2006a, auf s. 48).

Abb. 6. Ankara, Museum. Tönerne Krieger-Platte der "Gruppe II" aus Pazarl (Akurgal 1955, Taf. 46b).

Abb. 7. Ankara, Museum. Tönerne Krieger-Platte der "Gruppe IV" aus Pazarl (Akurgal 1955, Taf. 48a).

Abb. 8. Ankara, Museum. Tönerne Krieger-Platte der "Gruppe IV" aus Pazarlı (Işık 1991, Taf. 13c). 


\title{
ABKÜRZUNGSVERZEICHNIS
}

\author{
Akerström 1966 A. Akerström, Die architektonischen Terrakotten Kleinasiens (1966). \\ Akurgal 1955 \\ E. Akurgal, Phrygische Kunst (1955). \\ Akurgal 1966 \\ E. Akurgal, Orient und Okzident (1966). \\ Berndt 2002 \\ D. Berndt, Midasstadt in Phrygien. Eine sagenumwobenen Stätte im anatolischen \\ Hocbland (2002).
}

Bittel 1939 K. Bittel - A.M. Schneider - F.K. Dörner, "Archaeologische Funde aus der Türkei 1934-1938", $A A$ 1939, 94-207.

DeVries 2006a～K. DeVries, "Phryg Krallığ1, Midas'ın Ülkesi”, Arkeoatlas 5, 2006, 43-54.

DeVries 2006b K. DeVries, "Phryg Sanat1. Geometrik Doruk", Arkeoatlas 5, 2006, 58-61.

Işı1 1986

F. Işık, "Die Entstehung der frühen Kybelebilder Phrygiens und ihre Einwirkung auf die ionische Plastik”, ÖJh 57, 1986-1987 Beibl, 41-107.

Işık 1991 F. Işık, "Zur Entstehung der tönernen Verkleidungsplatten in Anatolien", AnatSt 41, 1991, 63-86.

Işık 2008 F. Işık, "Pişmiş Toprak Mimari Levhaların Frig Kökenine Yeni Bir Kanıt, in: T. Tarhan u.a. (Hrsg.), Mubibbe Darga Armağanı (2008) 275-281.

Prayon 1987 F. Prayon, Phrygische Plastik. Die früheisenzeitliche Bildkunst Zentral-Anatoliens und ibre Beziehungen zu Griechenland und zum Alten Orient (1987).

Roller 1999 L. E. Roller, In Search of God the Mother. The Cult of Anatolian Cybele (1999).

Sams 1989 G. K. Sams, "Sculpted orthostates at Gordion", in: K. Emre (Hrsg.), Anatolia and the ancient Near East. Studies in honour of Tahsin Özgü̧̈ (1989) 447-454.

Sams 1994 G. K. Sams, "Aspects of early Phrygian architecture at Gordion”, in: A. Çilingiroğlu - D. H. French (Hrsg.), Anatolian Iron Ages 3: the proceedings of the Third Anatolian Iron Ages Colloquium held at Van, 6-12 August $1990=$ Anadolu Demir Çağları 3: III. Anadolu Demir Çağları Sempozyumu bildirileri Van, 6-12 Ağustos 1990 (1994) 211-220.

Schefold 1950 K. Schefold, "Die Tonfriese von Pazarli", in: Deutsches Archäologisches Institut. Abteilung Istanbul (Hrsg.), Martin Schede zu seinem sechzigsten Geburtstag am 20. Oktober 1943 im Manuskript überreicht. Kleinasien und Byzanz: Gesammelte Aufsätze zur Altertumskunde und Kunstgeschichte, Istforsch 17 (1950) 137-148

Schefold $1964 \quad$ K. Schefold, Frühgriechische Sagenbilder (1964).

Strobel $2008 \quad$ K. Strobel, "Die Kulturelle und religiöse Entwicklung Altphrygiens I: Kontinuitäten - Brüche - Einflüsse”, AMS 61, 2008, 123-142.

Tüfekçi Sivas T. Tüfekçi Sivas, Eskişehir-Afyonkarahisar-Kütahya İl Sinırları İçindeki Phryg $1999 \quad$ Kaya Anttlar (1999).

Voigt 2006 M. M. Voigt, "Yassıhöyük. Başkent Gordion", Arkeoatlas 5, 2006, 28-34.

Wittke 2006 A.-M. Wittke, "Phrygia. Kayaların Efendileri”, Arkeoatlas 5, 2006, 10-24. 
Ein neuer Beleg zum phrygischen Ursprung der architektonischen Tonplatten

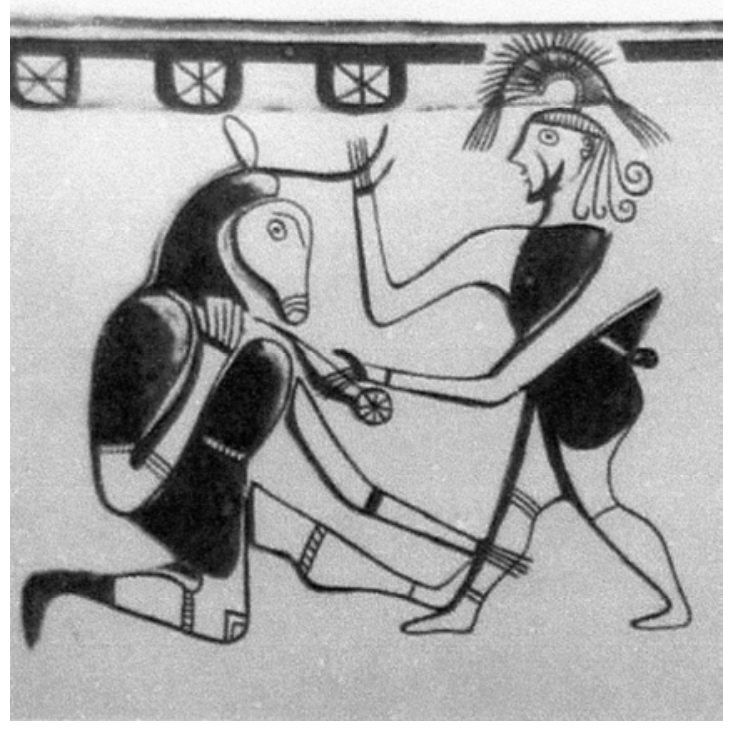

Abb. 1

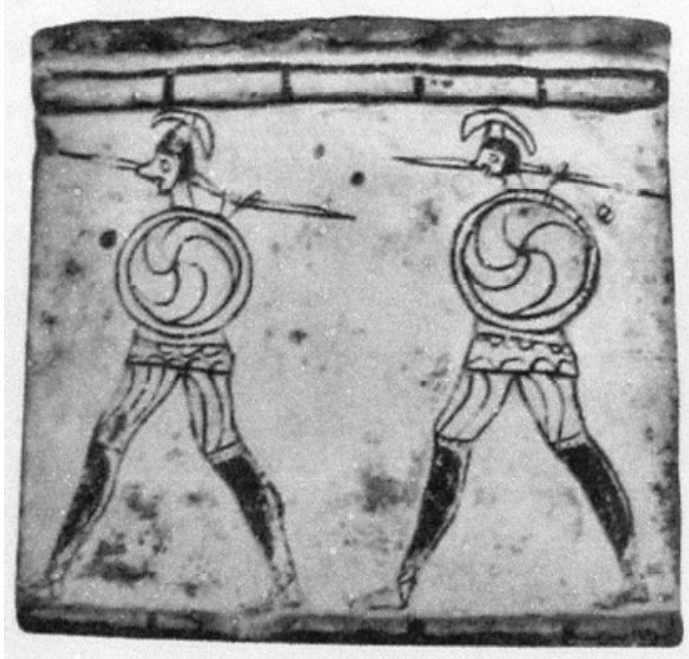

Abb. 3

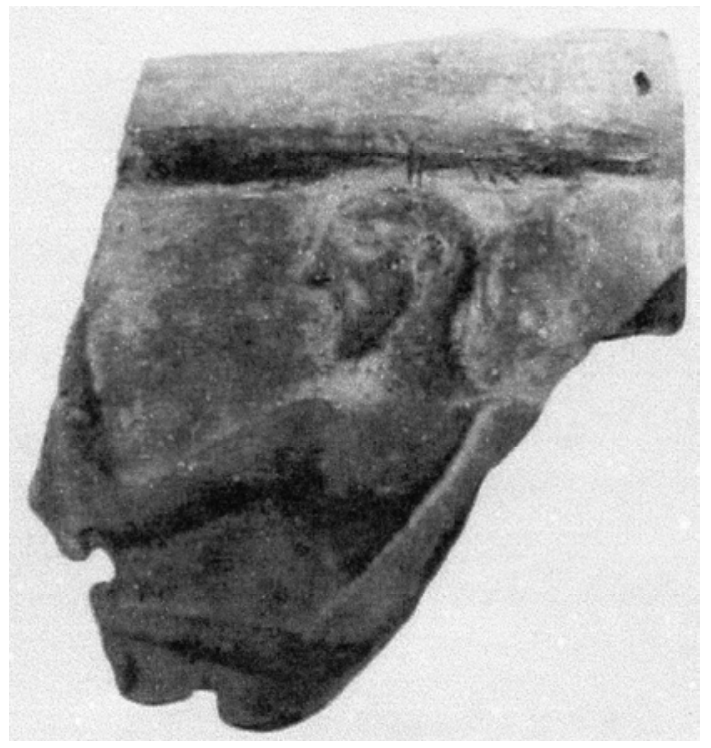

Abb. 2

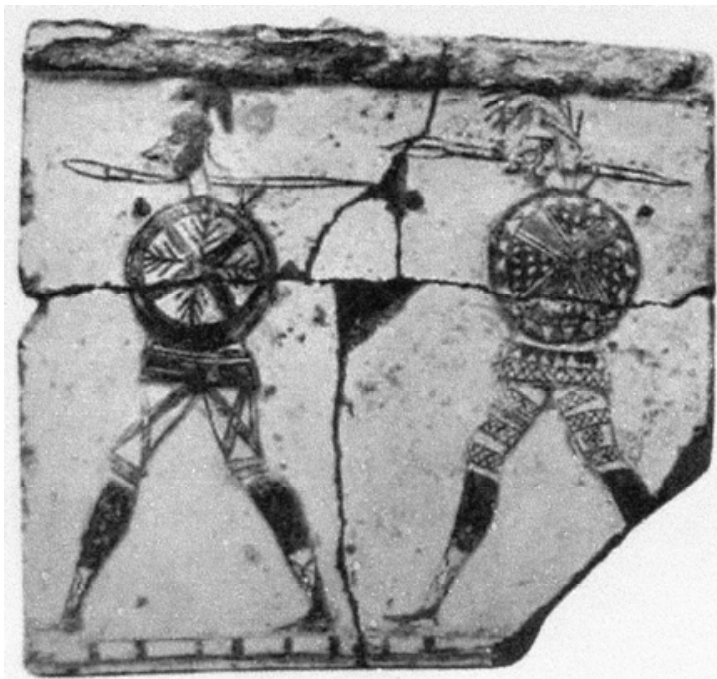

Abb. 4 


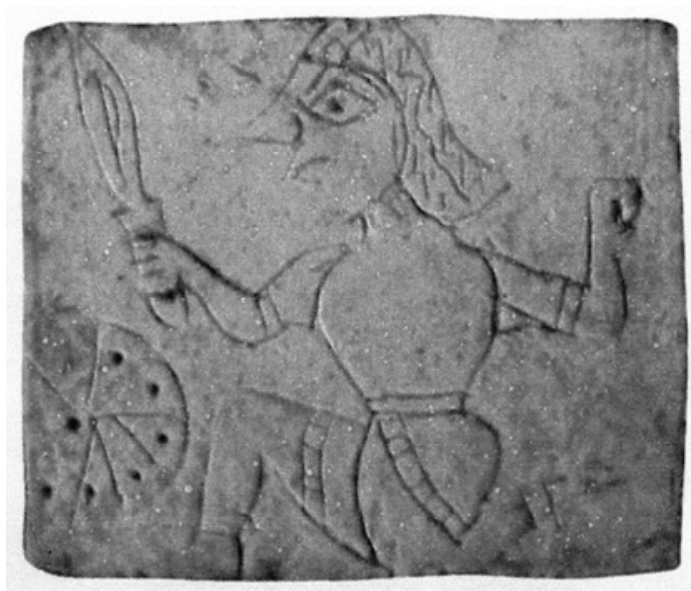

Abb. 5

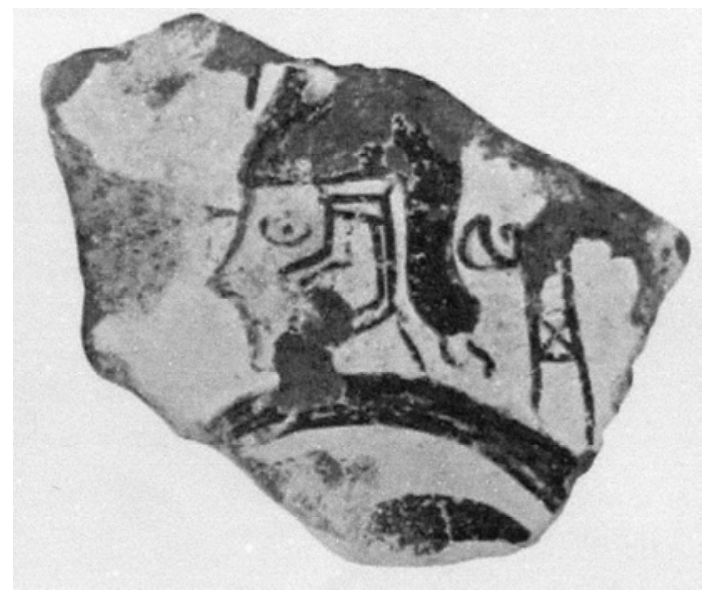

Abb. 7

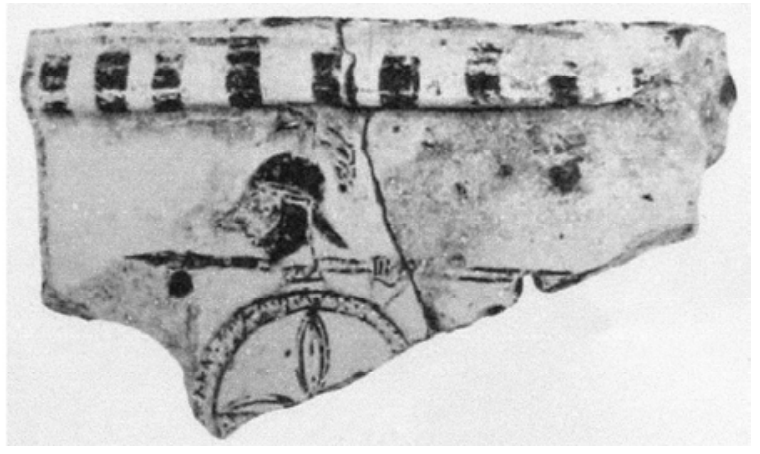

Abb. 6

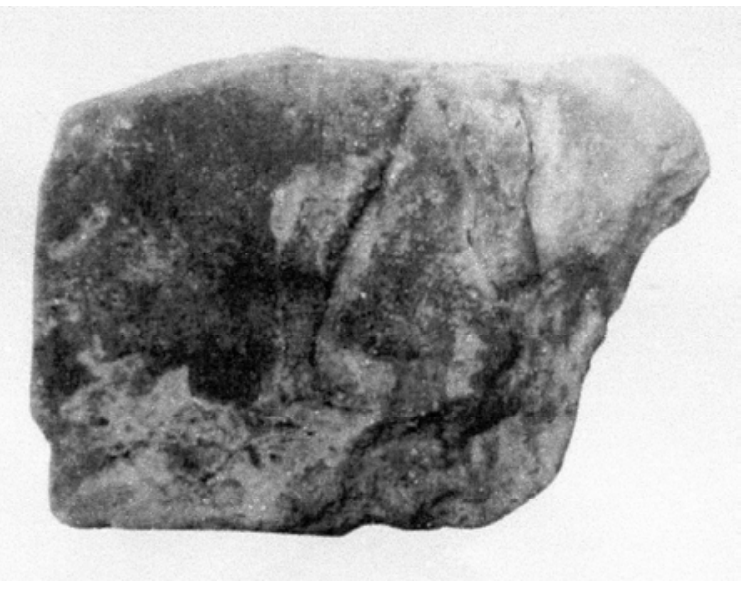

Abb. 8 